\title{
Knowledge, Awareness and Practice of Oral Premalignancy And Oral Cancer Screening and Diagnosis Among Medical Professionals - A Questionnaire Survey
}

\author{
Sam John Koshy ${ }^{1}$ and Madhulaxmi M² \\ ${ }^{1}$ Department of Oral \& Maxillofacial Surgery Saveetha Dental College and Hospitals, Saveetha \\ Institute of Medical And Technical Science, Saveetha University, Chennai- 600077, India \\ ${ }^{2}$ Professor Department of Oral \& Maxillofacial Surgery Saveetha Dental College and Hospitals, Saveetha \\ Institute of Medical And Technical Science, Saveetha University, Chennai- 600077, India
}

\section{ABSTRACT}

In developing countries like India, Oral cancer is considered to be the leading cause of mortality. Under such a scenario prompt detection and treatment of this devastating disease is a must. Detection of early oral changes and referrals of patients to specialists as and when required remain in the hands of general practitioners. . Lack of knowledge and awareness about oral premalignant lesions and oral cancer among medical students and doctors continue to be alarming as it dims the light on the necessity of knowledge about oral cancer and may contribute to delay in diagnosis and treatment. With this study, we aim to assess the knowledge, awareness and practice of medical professionals about oral premalignant lesions and oral lesions. A cross sectional, digital questionnaire based study was conducted among 377 medical professionals using 20 closed ended relevant questions. The collected data were consolidated and statistically analysed using IBM SPSS Version 20. The study revealed that $61.80 \%$ of participants were unaware of premalignant lesions and conditions out of which 65.25\% were not aware that these lesions and conditions can exist asymptomatically. $71.09 \%$ of participants were aware that oral cancer can be diagnosed at early stages. However, $68.7 \%$ of participants were unaware of the investigative procedures done for early detection. More than half the total participants (53.3\%) felt that they did not have sufficient knowledge concerning prevention of pre malignant oral lesion and conditions. This study concludes that more than $60 \%$ prospective medical professionals lack the knowledge about oral cancer and its diagnosis. This study hopes to shed light to the negligence of medical practitioners on a malignancy so relevant and yet left so unaware of. This study over all establishes the importance to improve the level of competency of the medical professionals in diagnosing and preventing oral premalignant lesions and oral cancer.

KEY WORDS: PREMALIGNANT LESION, CONDITION, ORAL CANCER.

\section{ARTICLE INFORMATION}

*Corresponding Author: madhulaxmi@saveetha.com

Received 1st Aug 2020 Accepted after revision 24th Sep 2020

Print ISSN: 0974-6455 Online ISSN: 2321-4007 CODEN: BBRCBA

Thomson Reuters ISI Web of Science Clarivate Analytics USA and Crossref Indexed Journal

$$
\begin{aligned}
& \text { Clarivate } \\
& \text { Analytics }
\end{aligned}
$$

NAAS Journal Score 2020 (4.31) SJIF: 2020 (7.728)

A Society of Science and Nature Publication,

Bhopal India 2020. All rights reserved.

Online Contents Available at: http//www.bbrc.in/

Doi: $h t t p: / / d x$.doi.org/10.21786/bbrc/13.8/127 


\section{INTRODUCTION}

A tumor is any abnormal proliferation of cells, which may be either benign or malignant. Benign tumors remain confined to the location of its origin, never invading the normal tissue of the surrounding nor causing metastasis by spreading to distant body sites. A malignant tumor on the other hand causes both invasion to the surrounding normal tissue and causes metastasis by spreading throughout the body by the circulatory or lymphatic systems. Malignant tumors are the only tumors referred to as cancer, and it is their ability to invade and metastasize makes it unpredictable and dangerous. (Cooper and Hausman, 2004). Oral cancer accounts for less than 5\% of the total incidence of all malignancies that occur in the human body, out of which, approximately half of the oral cancer patients die of their disease(Wright, 1994). Oral cancer is considered to be the leading cause of mortality in a developing country like India.

With a rich case bank established over 3 decades we have been able to publish extensively in our domain (Abdul Wahab et al., 2017; Eapen, Baig and Avinash, 2017; Patil et al., 2017; Jain and Nazar, 2018; J et al., 2018; Marimuthu et al., 2018; Wahab et al., 2018; Abhinav et al., 2019; Ramadorai, Ravi and Narayanan, 2019; Senthil Kumar et al., 2019; Sweta, Abhinav and Ramesh, 2019). Based on this inspiration we aim to assess the knowledge, awareness and practice of oral premalignancy and oral cancer screening and diagnosis among medical professionals.

Precancerous lesion or premalignant lesion is a tissue in the human body having undergone alterations morphologically in which oral cancer is more likely to occur than its apparently normal counterpart. These precancerous lesions include leukoplakia, erythroplakia, and the palatal lesions of reverse smokers.(Sarode, Sarode and Tupkari, 2014) Precancerous or premalignant condition is a generalized state associated with significantly increased risk of cancer. The precancerous conditions include submucous fibrosis, lichen planus, epidermolysis bullosa, and discoid lupus erythematosus. (Pindborg et al., 1997).

India, being a part of the Asian subcontinent, our attitude and resilience towards oral cancer needs to be retaught and our interest in knowledge, preventive measures and treatment modalities have to be in par with the scientific and medical advancements that occur that will help in the early diagnosis, prevention and rightful treatment protocol choice.(Brignardello-Petersen, 2020) India presents with a higher incidence of head and neck cancer contributing up to $7.8 \%$ of the global cancer burden and 8.33\% of global cancer deaths. More than 2 lakh new cases of head and neck cancers are diagnosed every year. (Prasad, 2014). This study aims to assess the knowledge, awareness and practice of medical professionals about oral premalignant lesions and oral lesions and hopefully improve the level of competency of the medical professionals in diagnosing and preventing oral premalignant lesions and oral cancer.

\section{MATERIAL AND METHODS}

A cross sectional, questionnaire based study was conducted among 377 medical professionals. A self designed, closed ended questionnaire containing 20 questions of relevance to understand the knowledge and awareness of medical professionals on oral cancer and premalignancy was framed. Questionnaire survey was conducted on Google forms where 377 medical professionals took part in the survey. The survey was conducted after informed consent from the participant. A total of 377 surveys were examined and evaluated with cross verification of data for the elimination of errors which could've aroused in the course of this study. Medical professionals who have graduated MBBS or are currently doing their internship were considered in this study. Inclusion of all available data with no form of sorting process has helped minimize sampling bias and stating applicable validity to the study.

Data was collected by means of Google forms. Medical students who had not joined the internship and other residents unwilling to participate were all excluded from the study. Out of a total of 392 participants, 15 participants were excluded from the study based on the exclusion criteria. A total of 377 patients were considered in this study. All the surveys were conducted with prior taken permission and consent making sure that the participant details remain confidential. Data was collected and verified by an external examiner and the statistical evaluation was done using IBM SPSS version 20. All results underwent statistical analysis at a confidence interval of 95\%.

\section{RESULTS AND DISCUSSION}

Out of a total of 392 participants who have taken part in the survey, 15 participants were excluded from the study as 7 did not answer the relevant questions and 8 participants were medical students in their early academic years. Out of the considered 377 participants, 20 self designed, closed ended questions were provided (Table 1). On consideration of the participant awareness of premalignant lesion and conditions, out of a total of 377 participants, 233 participants have replied No (61.8\%) whereas only 144 participants have replied Yes (38.2\%) [Figure 1] Considering the participants' awareness on whether premalignant lesions are always symptomatic, out of a total 377 participants, 246 have mentioned Yes, accounting for $65.3 \%$ whereas 131 participants have replied No (34.7\%). [Figure 2] Responding to the question whether oral cancer can be diagnosed in an early stage, out of a total of 377 participants, 268 have agreed with the statement (71.1\%) and 109 participants have disagreed with the statement (28.91\%).

[Figure 3] Out of a total of 377 participants to which the question, "do you know about the investigative procedures done for early detection of oral cancer", 259 participants have replied No (68.7\%) and 118 participants have replied Yes (31.3\%) [Figure 4] Considering the assessment of sufficient knowledge concerning the 
prevention and detection of pre malignant lesions and conditions, 201 participants out of the total 377 have said No (53.3\%) whereas 176 participants, accounting for $46.7 \%$ of the total participants have replied Yes. [Figure 5].

Table 1. Shows he survey questions and responses

Survey Questions

1.Are you aware about oral cancer?

2. . Do you know the various risk factors for oral cancer?

3.Are you aware of pre malignant lesions and conditions?

4. Are tobacco and alcohol the only etiological factors for oral cancer?

5. Do you think that the pre malignant lesions are always symptomatic?

6. Do you think that oral cancers can be diagnosed in early stage?

7. Oral cancer is a hereditary disease?

8. Does the risk of oral cancer increase with age?

9. Do you know about the investigative procedures done for the early detection of oral cancer?

10. Is oral cancer a preventable disease?

11. Lesions associated with smokeless tobacco generally resolve after discontinuation of these products.

12. Signs of lymph node - The most important characteristic of oral

cancer metastasis is a hard painless fixed lymph node?.

13. Early detection improves 5 year survival rates in oral cancer?

14. Annual oral cancer examinations should be provided for those of 40 years of age and above?

15. Do you feel that you have sufficient knowledge concerning prevention

and detection of pre malignant oral lesions and conditions?

16. Do you examine patients' oral mucosa routinely?

17. If your answer is No to the above question, do you screen the oral mucosa

if the patients are in high risk categories?

18. Do you record tobacco and alcohol use in personal history?

19. Do you take biopsy in patients with suspicious lesions?

20. Where would you refer a patient to dentist if you suspected an oral malignancy?

\begin{tabular}{|c|c|}
\hline $\begin{array}{c}\text { Response } \\
\text { YES }\end{array}$ & $\begin{array}{c}\text { Response } \\
\text { NO }\end{array}$ \\
\hline 278 & 99 \\
\hline 203 & 174 \\
\hline 144 & 233 \\
\hline 287 & 90 \\
\hline 246 & 131 \\
\hline 268 & 109 \\
\hline 143 & 194 \\
\hline 205 & 172 \\
\hline 259 & 118 \\
\hline 104 & 273 \\
\hline 212 & 165 \\
\hline 208 & 169 \\
\hline 294 & 83 \\
\hline 302 & 77 \\
\hline 176 & 201 \\
\hline 153 & 224 \\
\hline 218 & 6 \\
\hline 257 & 120 \\
\hline 249 & 128 \\
\hline 109 & 268 \\
\hline
\end{tabular}

Figure 1: Pie chart showing percentage of response of participants to the question 'are you aware of premalignant lesions and conditions?' where $61.80 \%$ of participants stated No (turquoise) and $38.20 \%$ participants stated Yes (violet).

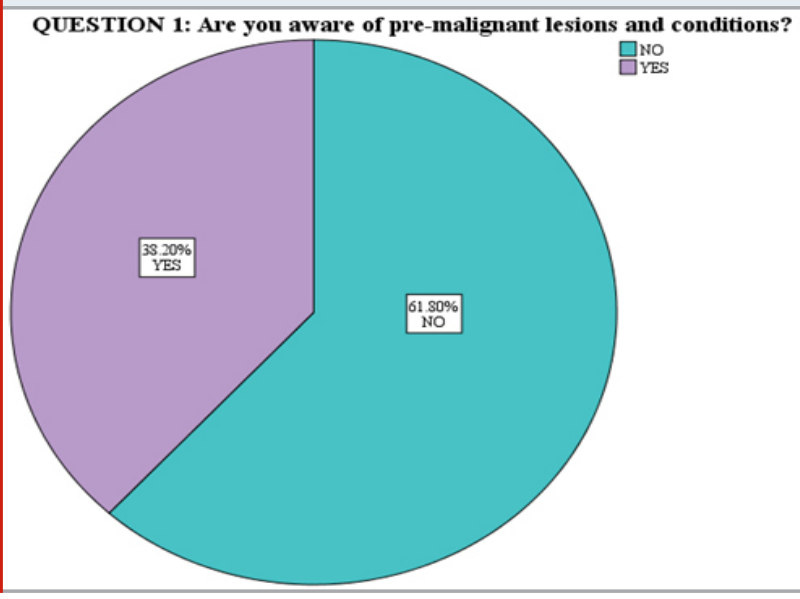

Figure 2: Pie chart showing percentage of response of participants to the question 'do you think pre malignant lesions are always symptomatic?'. 65.25\% of participants responded Yes(violet) and 34.75\% participants responded No (turquoise).

QUESTION 2: Do you think that the pre malignant lesions are always

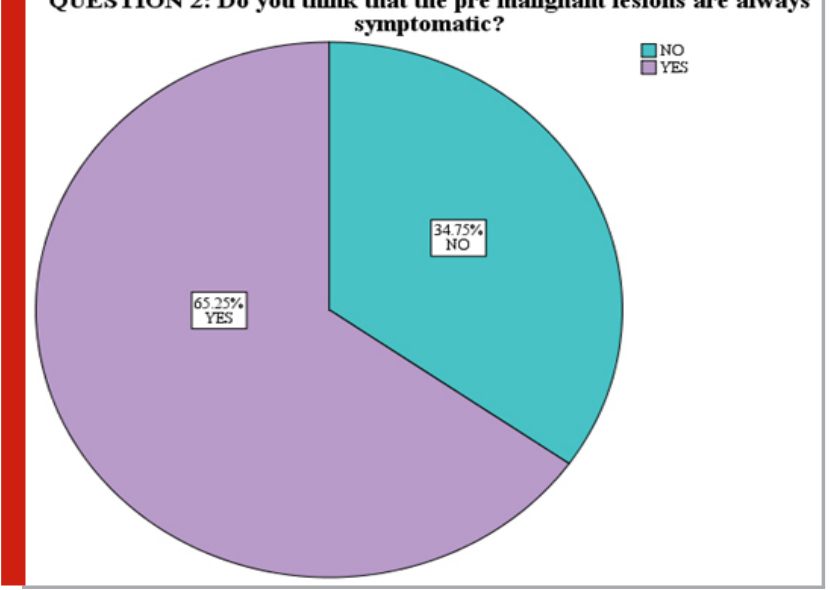


WHO in 1978 classified 'precancer' into 'lesions' and 'conditions'.(Organization and Others, 1973). Precancerous lesion or premalignant lesion is a tissue in the human body having undergone alterations morphologically in which oral cancer is more likely to occur than its apparently normal counterpart. (Sarode, Sarode and Tupkari, 2014) A state of disease associated with a significantly increased risk for the development of cancer is Precancerous or premalignant condition. (Pindborg et al., 1997). According to Lodi et al, pre malignant lesions include leukoplakia, erythroplakia, erythroleukoplakia, lichen planus, oral submucous fibrosis (Thomson, 2012)and premalignant conditions include advanced age, tobacco, smoking, or betel squid use, patients undergoing immunosuppression therapy, actinic keratosis, oral human papillomavirus infections, syphilis infections, discoid lupus erythematosus, xeroderma pigmentosa, dystrophic epidermolysis bullosa. (Lodi and Porter, 2007; Thomson, 2012; Liu et al., 2016). Early identification of the lesion and condition will help prevent its escalation to the status of malignancy.

Figure 3: Pie chart showing percentage of response of participants to the question 'do you think oral cancer can be diagnosed at an early stage?'. 71.09\% of participants responded Yes(violet) and 28.91\% participants responded No (turquoise).

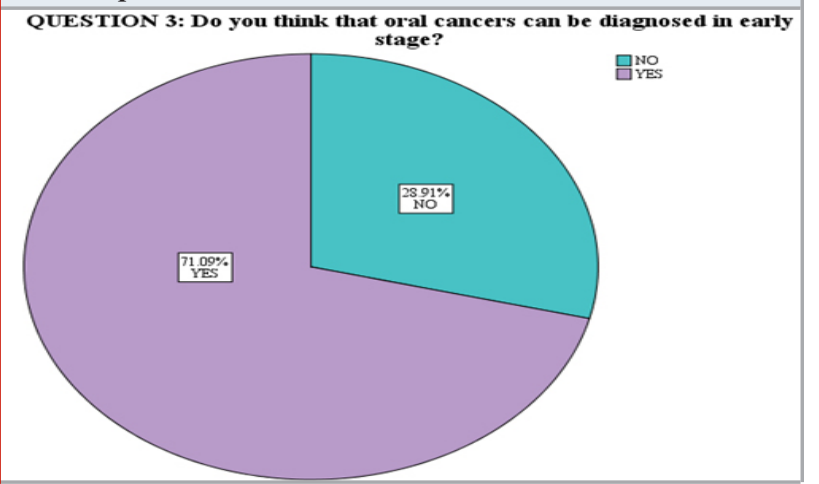

Figure 4: Pie chart showing percentage of response of participants to the question 'are you aware of the investigative procedures for the early diagnosis of oral cancer?' Out of a total of 377 participants, 259 participants have replied No (68.7\%) (turquoise) and 118 participants have replied Yes (31.3\%) (violet).

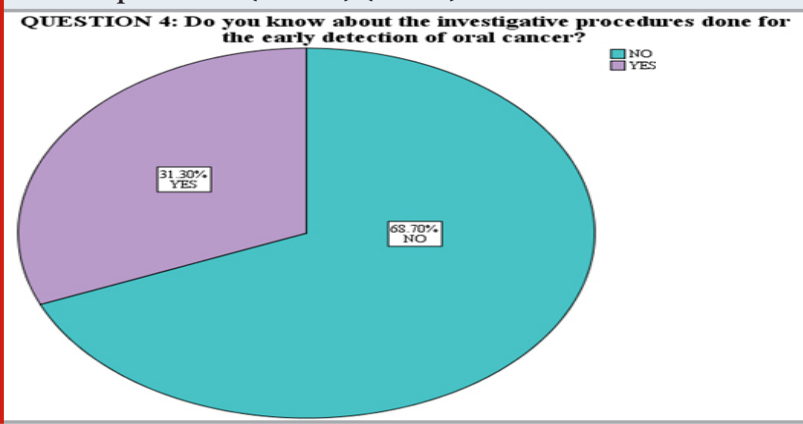

Figure 5: Pie chart showing percentage of response of participants to the question 'do you feel that you have sufficient knowledge concerning prevention and detection of pre malignant oral lesions and conditions?' 201 participants out of the total 377 have said No (53.3\%) (turquoise) whereas 176 participants, accounting for $46.7 \%$ of the total participants have replied Yes (violet).

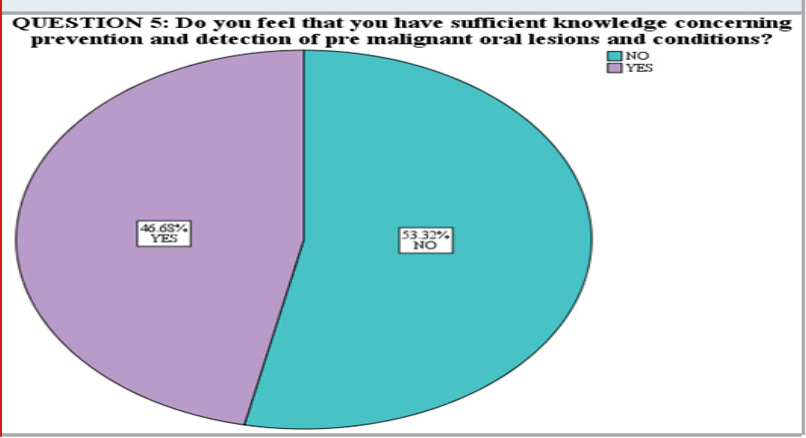

Medical practitioners who are in the first line of patient management, if educated on the signs of premalignant lesions and conditions and screening methods would help in early detection and diagnosis at a very primitive state. Screening tests done by medical practitioners should be highly applicable and acceptable to the general population, be cost effective, detect pre malignancy early at its natural stage, having a high positive predictive value and low false negatives making it highly sensitive.(Nair et al., 2012) Patients with a history of habits of smokeless or smoke form of tobacco, betel nut chewers and other deleterious habits, when approached for treatment to a medical practitioner should screen the patient for pre malignant lesions and conditions. Screening can be carried out by conventional oral examination using normal (incandescent) light and further by modern investigative and pathological tests. Oral screening is most effective in detecting premalignancy. Patients pertaining to other treatment with a positive history of deleterious habits should undergo a compulsory oral screening to rule out the presence of oral pre malignant lesions and conditions.

This will play a vital role in determining the five year life span of the patient which is crucial in understanding the outcome and prognosis of the disease and helps reduce mortality of the patients affected by it. Modern advancements in the area of imaging and radiology has helped shed light on the detection modalities such as oral cavity examination, supravital staining, oral cytology and optical technologies. Advanced optical technology such as spectroscopy, fluorescence spectroscopy, elastic scattering (reflectance) spectroscopy, Raman spectroscopy, fluorescence imaging, optical coherence tomography, narrow-band imaging, and multimodal optical imaging are of wide use to help identify and diagnose oral premalignant lesions and conditions at a very onset stage. (Steele and Meyers, 2011; Punyani and Sathawane, 2013; Yardimci et al., 2014).

Late diagnosis of oral cancers is usually made due to the lack of knowledge the clinician or the doctor has in 
identifying and diagnosing pre malignant lesions and conditions. Loss of chances to identify and characterise the disease are mainly of three in nature. Ignorance of the symptoms, lack of consciousness in patients, and a transient phase of negation are the factors that withhold the patient from visiting a doctor which are regarded as the "first time loss". The "second time loss" happens by the absence of medical and dental professionals' consciousness and absence of timely diagnosis. The final and "third time loss" is the period from the diagnosis to the start of therapy.(Thomas et al., 2003; Waal and van der Waal, 2009). Lack of knowledge and awareness about oral premalignant lesions and oral cancer among doctors of the medical profession continue to be alarming as it dims the light on the necessity of knowledge about oral cancer and may contribute to delay in diagnosis and treatment which may lead to an increased rate of mortality holistically. Therefore, knowledge is essential to help guide in early diagnosis, prevention and treatment of oral cancer.

\section{CONCLUSION}

Primary health care physicians play a very vital role in early diagnosis of oral premalignant lesions considering their progressive nature. The results of this survey study showed that more than $60 \%$ prospective medical professionals lack the knowledge about oral cancer and its diagnosis. To decrease the burden of this eminently preventable cancer, a multi faceted approach that integrates health education, tobacco and alcohol control, early detection, and early therapy is required. This study hopes to shed light on the need to improve the level of competency of the medical professionals in diagnosing and preventing oral premalignant lesions and oral cancer.

\section{ACKNOWLEDGEMENTS}

The authors express their sincere gratitude to all the respondents who had willingly participated in the study

Authors Contribution: Dr. Sam John Koshy carried out the retrospective study data retrieval, statistical analysis, sequence alignment and drafted the manuscript. Dr. Madhulaxmi M. conceived the study, participated in its design and coordination and helped draft the manuscript.

Conflicts of Interest: There were no conflicts of interest as defined by the authors.

\section{REFERENCES}

Abdul Wahab, P. U. et al. (2017) 'Risk Factors for Postoperative Infection Following Single Piece Osteotomy', Journal of maxillofacial and oral surgery, 16(3), pp. 328-332.

Abhinav, R. P. et al. (2019) 'The Patterns and Etiology of Maxillofacial Trauma in South India', Annals of maxillofacial surgery, 9(1), pp. 114-117.
Brignardello-Petersen, R. (2020) 'Professional oral care may decrease the incidence of oral mucositis in patients with advanced breast cancer', The Journal of the American Dental Association, p. e38. doi: 10.1016/j. adaj.2019.11.018.

Cooper, G. M. and Hausman, R. E. (2004) The Cell: A Molecular Approach. Sinauer Associates Incorporated.

Eapen, B. V., Baig, M. F. and Avinash, S. (2017) 'An Assessment of the Incidence of Prolonged Postoperative Bleeding After Dental Extraction Among Patients on Uninterrupted Low Dose Aspirin Therapy and to Evaluate the Need to Stop Such Medication Prior to Dental Extractions', Journal of maxillofacial and oral surgery, 16(1), pp. 48-52.

Jain, M. and Nazar, N. (2018) 'Comparative Evaluation of the Efficacy of Intraligamentary and Supraperiosteal Injections in the Extraction of Maxillary Teeth: A Randomized Controlled Clinical Trial', The journal of contemporary dental practice, 19(9), pp. 1117-1121.

J, P. C. et al. (2018) 'Prevalence and measurement of anterior loop of the mandibular canal using CBCT: A cross sectional study', Clinical implant dentistry and related research, 20(4), pp. 531-534.

Liu, D. et al. (2016) 'Non-Invasive Techniques for Detection and Diagnosis of Oral Potentially Malignant Disorders', The Tohoku journal of experimental medicine, 238(2), pp. 165-177.

Lodi, G. and Porter, S. (2007) 'Management of potentially malignant disorders: evidence and critique', Journal of Oral Pathology \& Medicine, pp. 63-69. doi: 10.1111/ j.1600-0714.2007.00575.x.

Marimuthu, M. et al. (2018) 'Canonical Wnt pathway gene expression and their clinical correlation in oral squamous cell carcinoma', Indian journal of dental research: official publication of Indian Society for Dental Research, 29(3), pp. 291-297.

Nair, D. R. et al. (2012) 'Oral cancer: Premalignant conditions and screening--an update', Journal of cancer research and therapeutics, 8 Suppl 1, pp. S57-66.

Organization, W. H. and Others (1973) 'Report of a meeting of investigators on the histological definition of precancerous lesions', Geneva: World Health Organization, 731.

Patil, S. B. et al. (2017) 'Comparison of Extended Nasolabial Flap Versus Buccal Fat Pad Graft in the Surgical Management of Oral Submucous Fibrosis: A Prospective Pilot Study', Journal of maxillofacial and oral surgery, 16(3), pp. 312-321.

Pindborg, J. J. et al. (1997) Histological Typing of Cancer and Precancer of the Oral Mucosa. Berlin, Heidelberg: Springer Berlin Heidelberg.

Prasad, L. (2014) 'Burden of oral cancer: An Indian 
scenario', Journal of Orofacial Sciences, p. 77. doi: 10.4103/0975-8844.143043.

Punyani, S. R. and Sathawane, R. S. (2013) 'Salivary level of interleukin-8 in oral precancer and oral squamous cell carcinoma', Clinical oral investigations, 17(2), pp. 517-524.

Ramadorai, A., Ravi, P. and Narayanan, V. (2019) 'Rhinocerebral Mucormycosis: A Prospective Analysis of an Effective Treatment Protocol', Annals of maxillofacial surgery, 9(1), pp. 192-196.

Sarode, S. C., Sarode, G. S. and Tupkari, J. V. (2014) 'Oral potentially malignant disorders: A proposal for terminology and definition with review of literature', Journal of oral and maxillofacial pathology: JOMFP, 18(Suppl 1), pp. S77-80.

Senthil Kumar, M. S. et al. (2019) 'Inflammatory pseudotumour of the maxillary sinus: clinicopathological report', Oral Surgery, 12(3), pp. 255-259.

Steele, T. 0. and Meyers, A. (2011) 'Early detection of premalignant lesions and oral cancer', Otolaryngologic clinics of North America, 44(1), pp. 221-9, vii.

Sweta, V. R., Abhinav, R. P. and Ramesh, A. (2019) 'Role of Virtual Reality in Pain Perception of Patients
Following the Administration of Local Anesthesia', Annals of maxillofacial surgery, 9(1), pp. 110-113.

Thomas, G. et al. (2003) 'Risk factors for multiple oral premalignant lesions', International journal of cancer. Journal international du cancer, 107(2), pp. 285-291.

Thomson, P. (2012) Oral Precancer: Diagnosis and Management of Potentially Malignant Disorders. John Wiley \& Sons.

Waal, I. van der and van der Waal, I. (2009) 'Potentially malignant disorders of the oral and oropharyngeal mucosa; terminology, classification and present concepts of management', Oral Oncology, pp. 317-323. doi: 10.1016/j.oraloncology.2008.05.016.

Wahab, P. U. A. et al. (2018) 'Scalpel Versus Diathermy in Wound Healing After Mucosal Incisions: A SplitMouth Study', Journal of oral and maxillofacial surgery: official journal of the American Association of Oral and Maxillofacial Surgeons, 76(6), pp. 1160-1164.

Wright, J. M. (1994) 'Oral precancerous lesions and conditions', Seminars in dermatology, 13(2), pp. 125-131.

Yardimci, G. et al. (2014) 'Precancerous lesions of oral mucosa', World journal of clinical cases, 2(12), pp. 866-872. 